\title{
Divergent effects of resistance training and anabolic steroid on the postsynaptic region of different skeletal muscles of aged rats
}

\author{
Walter Krause Neto ${ }^{\mathrm{a}, *}$, Wellington de Assis Silva ${ }^{\mathrm{a}}$, Adriano Polican Ciena ${ }^{\mathrm{b}}$, \\ Carlos Alberto Anaruma ${ }^{\mathrm{b}}$, Eliane Florencio Gama ${ }^{\mathrm{a}}$ \\ a Department of Physical Education, Laboratory of Morphoquantitative Studies and Immunohistochemistry, São Judas Tadeu University, São Paulo, SP, Brazil \\ b Department of Physical Education, Laboratory of Morphology and Physical Activity, São Paulo State University "Júlio de Mesquita Filho", Rio Claro, SP, Brazil
}

\section{A R T I C L E I N F O}

\section{Keywords:}

Strength training

Aging

Neuromuscular junction

Acetylcholine receptors

Peripheral nervous system

\begin{abstract}
A B S T R A C T
This study aimed to analyze the effects of resistance training associated with testosterone administration in the neuromuscular junction (NMJ) postsynaptic region of different skeletal muscle types of aged rats. Wistar rats were divided into: SEI - 20-months-old control, SEF - 24-months-old control, T - 20-months-old with testosterone, S - 20-months-old resistance trained and ST - 20-months-old with resistance training associated with testosterone propionate. All groups were submitted to familiarization and maximum load carrying testing (MLCT). The MLCT was applied before and after the resistance training (RT) period. RT $(6-8 \times /$ session with progressive loads of 50 to $100 \%, 3 \times$ /week and $120 \mathrm{~s}$ interval) was performed in ladder climbing for 15 weeks. The administration of testosterone propionate was performed $2 \times /$ week $(10 \mathrm{mg} / \mathrm{kg} / \mathrm{body}$ weight $)$. After euthanize, soleus and plantaris muscles were removed and prepared for histochemistry and cytofluorescence. T, S and ST significantly increased their maximum carrying load capacity compared to SEI and SEF ( $p<0.05$ ). For soleus postsynaptic region, ST had lower total and stained area than SEF $(p<0.05)$. For plantaris, the postsynaptic component of T was statistically larger than SEI $(p<0.05)$. For soleus histochemistry, T, S and ST groups showed the same magnitude of type I myofibers hypertrophy, thus statistically different from SEI and SEF $(p<0.05)$. The cross-sectional area of the type IIa myofibers of the ST was larger than SEF $(p<0.05)$. The volume density of type I myofibers show to be lower in ST than SEI $(p<0.05)$. As for type IIa myofibers, ST increased Vv [type IIa] compared to SEI and SEF $(p<0.05)$. For plantaris, T significantly hypertrophied type I myofibers compared to SEI and SEF $(p<0.05)$. S and ST demonstrated significant increases of type I myofibers compared to SEI and SEF $(p<0.05)$. As for type IIx myofibers, both S and ST showed myofibers larger than SEI $(p<0.05)$. However, only the ST had significant difference compared to SEF $(p<0.05)$. In conclusion, both therapies, alone or combined, have little effect on the morphology of the NMJ postsynaptic region of distinct muscles. Moreover, the three therapies are potentially stimulating for strength gains and muscle hypertrophy.
\end{abstract}

\section{Introduction}

Sarcopenia is a multifactorial process characterized by morphological, physiological and functional changes throughout the neuromuscular system. Recent studies have demonstrated that degeneration of the neuromuscular junction (NMJ) precedes alterations in the muscular profile leading to reductions in peripheral nerve components (Deschenes et al., 2010; Jang and Van Remmen, 2011; Sakita et al., 2016). Initially, the process of reinnervation compensates denervation, and with the continuum of this neurogenic process, more myofibers will become permanently unweaned. The loss of some muscle fibers directs the change in the functional profile, possibly altering the neuromuscular pattern. Yet this change may be more striking for muscles predominantly composed of fast-twitch myofibers than for those with slow contraction rates (Deschenes et al., 2015). Despite this, Deschenes et al. (Deschenes et al., 2010) quote that aging-related denervation occurs prior to muscle atrophy, and maintenance or increase of neuromuscular activity (eg. exercise) may delay the onset of predominance of denervation and sarcopenia related to aging.

Studies have documented that endurance training over a period of a few weeks may alter several parameters of the NMJ architecture, including its size and length, synaptic vesicle and acetylcholine receptors (AChRs) dispersion, as well as branching of nerve endings. However, the responsiveness of NMJ from aged muscles to resistance training (RT), which implies brief intervals of moderate to high-intensity neuromuscular activity, has been little investigated. Deschenes et al.

\footnotetext{
* Corresponding author at: Rua Taquari, 546, Mooca Unit, P.O. box 03166-000, São Paulo, SP, Brazil.

E-mail address: wild_krause@hotmail.com (W. Krause Neto).
} 
(Deschenes et al., 2015) applied a 7 week RT protocol revealing that remodeling of NMJ was evident only at the postsynaptic end-plate region of soleus type II myofibers, without any effects in the aged plantaris muscle.

Testosterone and its pharmacological derivatives, such as anabolic androgenic steroids (AAS), are potent regulators of muscle mass and have a stimulating trophic effect on muscle tissue in various populations (Carson et al., 2002). AAS are synthetic substances derived from the molecule of the hormone testosterone and seek to maximize the anabolic effects on tissues by increasing the half-life of the hormonal action. The use of AAS as a form of treatment of chronic disorders can maintain or increase the mass and muscular strength of young and old individuals. Thus, muscle protein synthesis in the elderly is increased followed by the use of AAS. Nevertheless, this effect seems to depend on the dosage, steroid type and application schedule (Krause Neto et al., 2015). AASs act on skeletal muscle and may act synergistically with resistance training (RT) in maintaining or increasing strength and muscle mass during aging. However, the effects of using testosterone on specific peripheral nervous system components (such as NMJ), which would help to explain outcomes seen in several human studies, are still scarce. Cappello et al. (Cappello et al., 2012) presented evidence indicating that the use of AAS can prevent or decelerate structural changes in the NMJ of rats with neurogenerative disease.

Recent studies have presented evidence that suggests more expressive results by associating resistance training with the use of testosterone therapies in the elderly (Hildreth et al., 2013; Kvorning et al., 2013). However, it is not known whether this association also exerts some type of modulation on the neuromuscular junction morphology. In single evidence, Cavalcante et al. (Cavalcante et al., 2004) demonstrated a toxic effect of nandrolone decanoate on synaptic transmission in sedentary rats. However, such an effect was masked by the trained group. This fact gives rise to a probable concomitant effect between the two interventions.

Thus, the aim of the present study was to analyze the effects of resistance training associated with administration of exogenous testosterone in the NMJ postsynaptic region of different skeletal muscle types of aged rats.

\section{Materials and methods}

This study was authorized by the Committee on Ethics in Animal Use (CEAU - Protocol 001/2013) and carried out at the Laboratory of Morphoquantitative Studies and Immunohistochemistry of the São Judas Tadeu University (LEMI-USJT), Laboratories of Histology and Morphology and Physical Activity of the Institute of Biosciences of the São Paulo State University "Júlio de Mesquita Filho" (UNESP-Rio Claro) and the Laboratory Headquarters of the National Institute of Photonics Applied to Cell Biology of the State University of Campinas (INFABICUNICAMP).

Thirty Wistar male rats (Rattus norvegicus), 20 months of age, were divided into five groups as described below: SEI - 20 months-old that were used as initial control $(n=6)$; SEF - 24 months-old which remained as final control of the procedures $(n=6)$; T - 20 months-old which received injections of testosterone propionate $(n=6)$; S 20 months-old which underwent resistance training $(n=6)$ and ST 20 months-old which underwent resistance training and injections of testosterone propionate $(n=6)$.

During the experimental period, the animals were housed in polypropylene boxes kept in controlled environments of temperature $\left(22^{\circ} \mathrm{C}\right)$ and illumination (12 h light and 12 dark hours). For all groups were provided commercial reference food for rats and water ad libitum.

\subsection{Resistance training protocol}

For resistance training, we adapted the accommodation room by reversing the light-dark cycle (lights programmed to remain off from
7 o'clock in the morning to 7 o'clock in the evening and on from 7 o'clock to 7 o'clock in the morning). This procedure was done to train all animals at the active period (dark). Training sessions were done between $11 \mathrm{a} . \mathrm{m}$. and $4 \mathrm{p} . \mathrm{m}$. of the scheduled days using ladder climbing (LC) model. The equipment was constructed in wood with steps of iron, $110 \mathrm{~cm}$ of height, $80^{\circ}$ of inclination and distance of $2 \mathrm{~cm}$ between each step (Hornberger and Farrar, 2004). At the top of the equipment was placed a plastic box to accommodate the animals in the interval between climbs.

During adaptation, each animal climbed the ladder three times, starting from distinct positions at intervals of $60 \mathrm{~s}$, during five sessions (Monday to Friday) and without any additional burden attached to the animal's tail. Animals did not had any help or reward to climb the equipment. After this period, the animals of all groups underwent the maximum loaded carrying test.

All rodents performed the maximum loaded carrying test (MLCT) in two sessions. The option to perform two sessions was motivated by the existence of evidences, which demonstrated that sedentary individuals need more than one session of maximum load tests to reduce the initial strength gain bias by improving their neural coordination (Dias et al., 2005; Krause Neto et al., 2016).

The MLCT consisted about the animal climb the ladder as many times as possible from a given initial load, adding to each successful attempt a new percentage. Each rodent climbed the ladder twice, without any additional load, with one-minute interval between each climb. Then, was calculated the initial loading from $50 \%$ of the animal's body weight (BW), with subsequent increases of $10 \%$ of the BW being made at each successful climb. The test was conducted until the animal was unable to climb the ladder for at least two consecutive attempts (failure). Between attempts, animals had an interval of two minutes. From traditional protocol of Hornberger and Farrar (Hornberger and Farrar, 2004) an initial load of $75 \%$ of BW was used. However, due to advanced age of our rodents ( 20 months) we adapted the initial loads to $50 \%$ of the BW.

After MLCT, we submitted S and ST groups to the RT protocol (Lee et al., 2004; Harris et al., 2010) during fifteen weeks (45 sessions). In the first session, the rats climbed the ladder twice at 50, 75 and $100 \%$ of the final load achieved in the MLCT. If the animal could climb the ladder twice with $100 \%$, an extra $30 \mathrm{~g}$ was added for two more climbs. During the subsequent sessions, relative new percentages were calculated from maximum loads reached in the previous session. However, if the animal could not complete the protocol with eight maximum climbs, the same loads were maintained for the next training session. Each rodent had a two-minute interval between each climb. The RT was performed three times a week on alternate days (Mondays, Wednesdays and Fridays).

To increase the loads we used lead weights attached by a musket and tape, tied to the proximal region of the animal's tail. The body weight of all animals was measured once a week (Tuesdays). For this, a semi-analytical balance of the GEHAKA BG 100 brand was used. The initial and final body weight of each group, together with the soleus (SL) and plantaris (PL) ratios, were tabulated at the end of experimentation.

After training, we analyzed the following parameters: absolute total load $(\mathrm{kg})$ and maximum carrying load capacity. The absolute total load was calculated by summing loads carried during the entire training period.

\subsection{Anabolic steroid administration}

The PERINON ${ }^{\circledR}$ anabolic steroid from the Perini laboratory in a $100 \mathrm{ml}$ vial containing $200 \mathrm{mg} / 20 \mathrm{ml}$ of testosterone propionate (TP) was administrated by intraperitoneal injection, twice a week (Tuesdays and Fridays), starting from the first day of the experiment in the $\mathrm{T}$ and ST groups. The animals received the injections from the age of 20 months, shortly after the MLCT, until the scheduled date of 
euthanasia. The duration of treatment was concomitant with the RT of the S and ST groups, i.e. fifteen weeks.

There is no standard dosage of weekly application of this hormone to rodents. So, was used the dosage of $10 \mathrm{mg} / \mathrm{kg} \mathrm{BW} /$ week. It is known that this dosage is sufficient to stimulate the increase of bone mineral density and growth hormone in rats (Fitts et al., 2004). In addition, previous data from our laboratory demonstrated a significant muscular hypertrophy and low level of hepatic side effect with this dosage in middle-aged rats (Tanasov et al., 2014). We weighted the animals every Tuesday to recalculate the application dosage.

\subsection{Euthanasia, collection and preparation of the material for analysis}

The animals were euthanized using the $\mathrm{CO}_{2}$ inhalation, according to the schedule of each experimental group. After euthanasia, we removed SL and PL muscles, preparing them for cytofluorescence and histochemistry techniques. The SL and PL muscles were chosen according to their predominance of muscle fiber and muscular recruitment during the climbing training. According to Deschenes et al. (Deschenes et al., 2000), the visualization of NMJ in muscles with more homogeneous characteristics is better, since in more heterogeneous muscles, the NMJs present different characteristics. The SL muscle is a predominantly oxidative muscle composed of a majority of type I myofibers. Contrarily, PL muscle is glycolytic and predominantly formed of faster contraction myofibers (type II). Thus, an incision was made in the posterior portion of the right knee of the animal up to his ankle to expose SL and PL muscles. After the muscles are exposed, cleaned of fat and connective tissue, we quickly prepare them for specific analyses of cytofluorescence and light microscopy.

\subsection{Cytofluorescence}

For this study, we used the INFABIC-UNICAMP inverted LSM780NLO Zeiss Microscope. The preparation of the slides took place in the Laboratory of histology from UNESP-Rio Claro.

After the extraction and cleaning of the SL and PL muscles, we removed the middle third of each muscle, leaving them in nitrogen and then kept in a cold tank at $-80^{\circ} \mathrm{C}$. In order to visualize the postsynaptic component of each $\mathrm{NMJ}$, we made longitudinal cross-sections of $100 \mu \mathrm{m}$ of thickness using cryotome at $-20^{\circ} \mathrm{C}$. To avoid tissue contraction, the slides were pretreated in $3 \%$ ethylenediaminetetraacetic acid solution. Sections were washed six times for $5 \mathrm{~min}$ each in phosphate buffered saline (PBS) containing 1\% bovine serum albumin (BSA). Then the sections were incubated overnight $(16 \mathrm{~h})$ in a humidified chamber at $4{ }^{\circ} \mathrm{C}$ in a solution containing $\alpha$-bungarotoxin conjugated rhodamine (BTX; Molecular Probes, Eugene, OR-T-1175), diluted 1: 600 in PBS. After this period, the sections were given a final wash $(6 \times 5 \mathrm{~min})$ before being lightly coated with Prolong (Molecular Probes, Eugene, OR-P10144) and having their coverslips applied. The slides were then encoded with respect to the intervention group in order to allow assessment of the morphology of the motor end-plate. Thereafter, they were stored at $-20{ }^{\circ} \mathrm{C}$ until analysis.

For morphometry, 12 to 15 frames, containing one end-plate each with final magnification of $1000 \times$ were captured. In the analysis of the postsynaptic compound of NMJ (Fig. 1), we measured the total perimeter $(\mu \mathrm{m})$, or the length covering the entire end-plate composed of groups of stained and non-stained receptors regions interspersed within these groups, perimeter stained $(\mu \mathrm{m})$, or the composite length of tracings around groups of individual receptors, the total area $\left(\mu \mathrm{m}^{2}\right)$, which includes the receivers stained together with uncolored regions interspersed between groups of receptors, the stained area $\left(\mu \mathrm{m}^{2}\right)$ or the areas occupied by groups and the dispersion of the end-plates, which was evaluated by dividing the stained area of the terminal plate by its total area and multiplying the value by 100 (Deschenes et al., 2013a). To do this, we use the software axiovision 4.8.

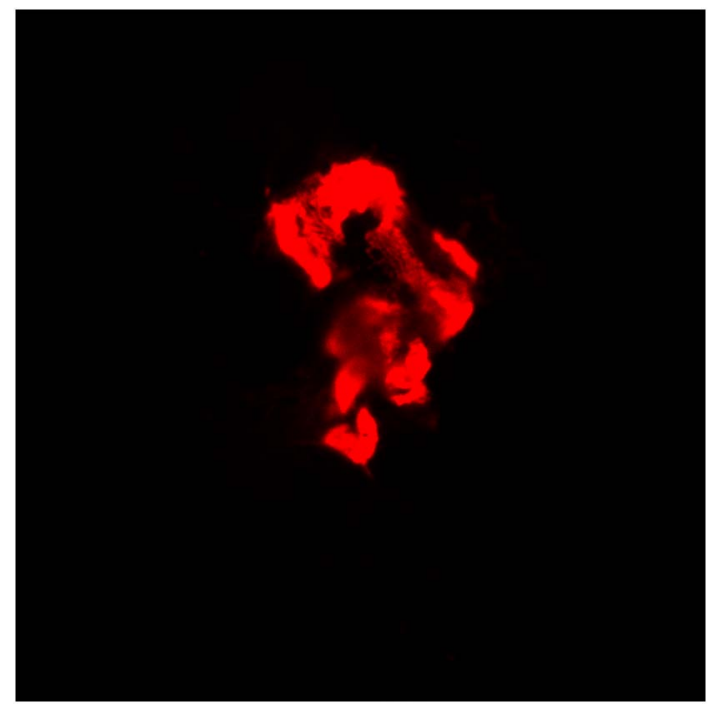

Fig. 1. Cytofluorescent labeling image for postsynaptic acetylcholine receptors. Confocal microscopy. Image stained with rhodamine $\alpha$-bungarotoxin. $1000 \times$ amplification.

\subsection{Histochemistry}

The SL and PL muscles were cryophilized in liquid nitrogen and kept at $-80{ }^{\circ} \mathrm{C}$ until analysis. The assembly and preparation of the slides were done at the Laboratory of Morphology and Physical Activity of UNESP-Rio Claro.

After sectioning the tissue to cryo- $20{ }^{\circ} \mathrm{C}$ in the thickness of $8 \mu \mathrm{m}$, the sections were incubated for $30 \mathrm{~min}$ at $37^{\circ} \mathrm{C}$ in a solution containing $10 \mathrm{mg}$ of ATP dissolved in 2 drops of distilled water added with $10 \mathrm{ml}$ of more glycine/ $\mathrm{NaCl}$ buffer $\mathrm{CaCl}^{2}$ and hitting the $\mathrm{pH}$ to 9.4 added with DDT. Then, we washed the cuts in distilled water and incubated for $2 \mathrm{~min}$ in $2 \%$ cobalt chloride for 3 times. Again, we washed in distilled water and dehydrate in ascending alcohol series $(70 \%, 90 \%, 95 \%$ and $100 \%)$ and brighten in xylol. Finally, we set up in Balsamo of Canada. For the method at $\mathrm{pH} 4.3$ and 4.6 , we preincubated the sections in $0.1 \mathrm{M}$ sodium acetate buffer with $10 \mathrm{mM}$ EDTA for $10 \mathrm{~min}$ at $4{ }^{\circ} \mathrm{C}$ at $\mathrm{pH} 4.3$ and 4.6. Wash with distilled water and incubate for $2 \mathrm{~min}$ in $2 \%$ cobalt chloride for 3 times. Again, we washed in distilled water and dehydrated in ascending alcohol series (70\%, 90\%, 95\% and 100\%), lighten it in xylol. Finally, we set up in Canada balsam (Fig. 2).

For skeletal muscles stereology (Vv), the slides corresponding to each group were photographed under a light microscope with a final magnification of $100 \times$. For this analysis, 30 photographs of each group

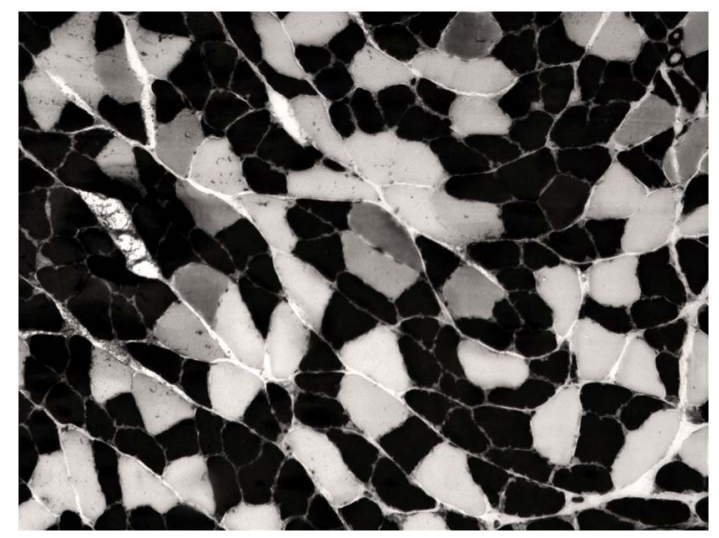

Fig. 2. Sample of myofibers from the soleus muscle of Wistar rats histochemically stained for myosin ATPase following acidic preincubation. Darker myofibers are type I, clearer are type IIa myofibers, and intermediates are type IIx myofibers. Light microscopy. Original magnification of $100 \times$. 
Table 1

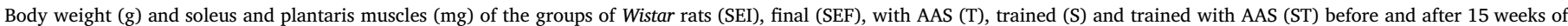
experimentation.

\begin{tabular}{|c|c|c|c|c|c|c|c|}
\hline \multirow[b]{2}{*}{ Variables } & \multicolumn{5}{|l|}{ Groups } & \multirow[b]{2}{*}{$\mathrm{F}$} & \multirow[b]{2}{*}{$p$ value } \\
\hline & SEI & SEF & $\mathrm{T}$ & $\mathrm{S}$ & ST & & \\
\hline Initial body weight & $590,71 \pm 60,17$ & $683,33 \pm 78,46^{\mathrm{a}}$ & $628,75 \pm 37,3$ & $675,63 \pm 49,38^{\mathrm{a}}$ & $565 \pm 62,65^{\mathrm{b}, \mathrm{d}}$ & 4673 & 0,002 \\
\hline Final body weight & - & $611,67 \pm 116,18$ & $536,25 \pm 49,19$ & $614,37 \pm 55^{*}$ & $481 \pm 32,48^{*, \mathrm{~b}, \mathrm{~d}}$ & 5197 & 0,007 \\
\hline Soleus weight & $293,14 \pm 55,57$ & $268,29 \pm 49,89$ & $281,29 \pm 29,65$ & $296,29 \pm 32,38$ & $266,8 \pm 33,48$ & 1053 & 0,404 \\
\hline Soleus ratio & $0,5 \pm 0,09$ & $0,44 \pm 0,08$ & $0,53 \pm 0,05$ & $0,48 \pm 0,05$ & $0,56 \pm 0,07$ & 1982 & 0,107 \\
\hline Plantaris weight & $518,71 \pm 54,55$ & $457,71 \pm 50,54$ & $490,14 \pm 42,73$ & $590,14 \pm 61,29^{\mathrm{b}, \mathrm{c}, \mathrm{d}}$ & $452,6 \pm 25,62^{\mathrm{d}}$ & 8200 & 0,000 \\
\hline Plantaris ratio & $0,88 \pm 0,09$ & $0,75 \pm 0,08^{\mathrm{a}}$ & $0,91 \pm 0,08^{\mathrm{b}}$ & $0,96 \pm 0,09^{b}$ & $0,94 \pm 0,05^{\mathrm{b}}$ & 5884 & 0,001 \\
\hline
\end{tabular}

Values are presented as mean \pm standard deviation.

ratio $=$ muscle weight/body weight.

${ }^{a}$ Indicates a significant difference in relation to SEI $(p \leq 0.05)$.

${ }^{\mathrm{b}}$ Indicates a significant difference in relation to SEF $(p \leq 0.05)$.

${ }^{\mathrm{c}}$ Indicates significant difference in relation to $\mathrm{T}(p \leq 0.05)$.

${ }^{\mathrm{d}}$ Indicates a significant difference in relation to $\mathrm{S}(p \leq 0.05)$.

${ }^{*}$ Indicates a significant difference between the PRE versus POST moments $(p \leq 0.05)$.

were taken. The $\mathrm{Vv}$ of type I ( $\mathrm{Vv}[\mathrm{I}]$ ), IIa ( $\mathrm{Vv}$ [IIa]), IIx ( $\mathrm{Vv}$ [IIx]) myofibers and interstitium ( $\mathrm{Vv}$ [inters]) of each muscle were calculated. In these procedures, we used the software image $\mathrm{J}$.

For morphometry, slides containing transversal sections of the SL and PL muscles were analyzed. For this, we analyzed 30 photographs of each group with final magnification of $400 \times$. Measurements were made in software axiovision version 4.8 coupled to the light microscope (Zeiss). In each slide was possible to measure the cross-sectional area $\left(\mu \mathrm{m}^{2}\right)[\mathrm{CSA}]$ of myofibers type I, IIa and IIx.

\subsection{Statistical analysis}

All data are presented by mean and standard deviation. For statistical comparison between the different groups, one-way ANOVA (post hoc Tukey) was used. For comparison of the PRE versus POST results of the same group Student's $t$-test was calculated for dependent samples (MLCT pre to post). To compare the same parameters among the trained groups, we used $t$-test for independent samples (absolute loads). For the statistical calculations we used SPSS software version 21.0 and set the level of significance at $p \leq 0.05$. To demonstrate the statistical differences, we used fixed signals in order to facilitate the reading and comparison of the results.

\section{Results}

\subsection{Training parameters}

$\mathrm{S}$ and ST groups trained during 45 sessions. Experimental groups T, S and ST significantly increased their maximum carrying load capacity
(43\%, 81\% and 66\%, respectively) compared to SEI and SEF $(p<0.05)$. Absolute training loads were not different between $\mathrm{S}$ and ST groups (154.3 kg and $147.1 \mathrm{~kg}$, respectively).

\subsection{Body weight and muscle mass}

At the end of the experiment, the ST group had body weight significantly lower than SEF and $S(p \leq 0.05)$. Analyzing the PRE to POSTintervention moments, we identified that T (14.7\%), S (9.1\%), and ST (14.8\%) had a significant reduction in body weight ( $p \leq 0.05)$.

The weight of SL and PL muscles, together with their respective ratios, presented different responses at the end of the study. The SL muscle was not influenced by either aging or the administered interventions.

Comparing the results of the interventions, we found that $\mathrm{T}, \mathrm{S}$ and ST showed significantly higher ratios than $\operatorname{SEF}(p \leq 0.05)$. Data are presented in Table 1.

\subsection{Postsynaptic regions}

\subsubsection{Soleus}

Data are presented in Table 2. The ST group had lower total and stained area than SEF $(p<0.05)$. For all other parameters no statistical differences were found (Fig. 3).

\subsubsection{Plantaris}

Data are presented in Table 3. The administration of TP induced an increase in total and stained area and total perimeter (Fig. 4). Thus, the postsynaptic component of $\mathrm{T}$ group was statistically larger than SEI

Table 2

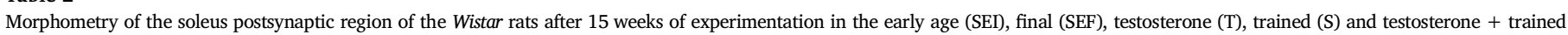
(ST) groups.

\begin{tabular}{|c|c|c|c|c|c|c|c|}
\hline \multirow[b]{2}{*}{ Variables } & \multicolumn{5}{|l|}{ Groups } & \multirow[b]{2}{*}{$\mathrm{F}$} & \multirow[b]{2}{*}{$p$ value } \\
\hline & SEI & SEF & $\mathrm{T}$ & $S$ & ST & & \\
\hline \multicolumn{8}{|l|}{ Non-stained receptors } \\
\hline Total perimeter $(\mu \mathrm{m})$ & $136,94 \pm 18,5$ & $136,48 \pm 24,32$ & $117,34 \pm 40,09$ & $119,97 \pm 8,07$ & $102,48 \pm 38,16$ & 1863 & 0,136 \\
\hline Total area $\left(\mu \mathrm{m}^{2}\right)$ & $890,35 \pm 267,54$ & $1085,03 \pm 299,5$ & $795,03 \pm 622,27$ & $888,24 \pm 167,09$ & $528,81 \pm 316,73^{\mathrm{a}}$ & 2835 & 0,036 \\
\hline \multicolumn{8}{|l|}{ Stained receptors } \\
\hline Perimeter $(\mu \mathrm{m})$ & $178,21 \pm 33,37$ & $188,96 \pm 24,04$ & $161,7 \pm 86,16$ & $189,62 \pm 20,88$ & $159,07 \pm 66,22$ & 0,948 & 0,467 \\
\hline Area $\left(\mu \mathrm{m}^{2}\right)$ & $550,13 \pm 245,85$ & $677,6 \pm 238,59$ & $447,77 \pm 339,99$ & $539,12 \pm 128,67$ & $278,95 \pm 166,2^{\mathrm{a}}$ & 4053 & 0,007 \\
\hline Dispersion (\%) & $61,44 \pm 15,75$ & $61,27 \pm 5,43$ & $56,43 \pm 6,79$ & $60,53 \pm 8,61$ & $53,05 \pm 4,81$ & 2015 & 0,110 \\
\hline
\end{tabular}

Values are presented as mean \pm standard deviation.

Dispersion $=$ stained area/total non-stained area $\times 100$.

${ }^{a}$ Indicates significant difference in relation to SEF $(p \leq 0.05)$. 

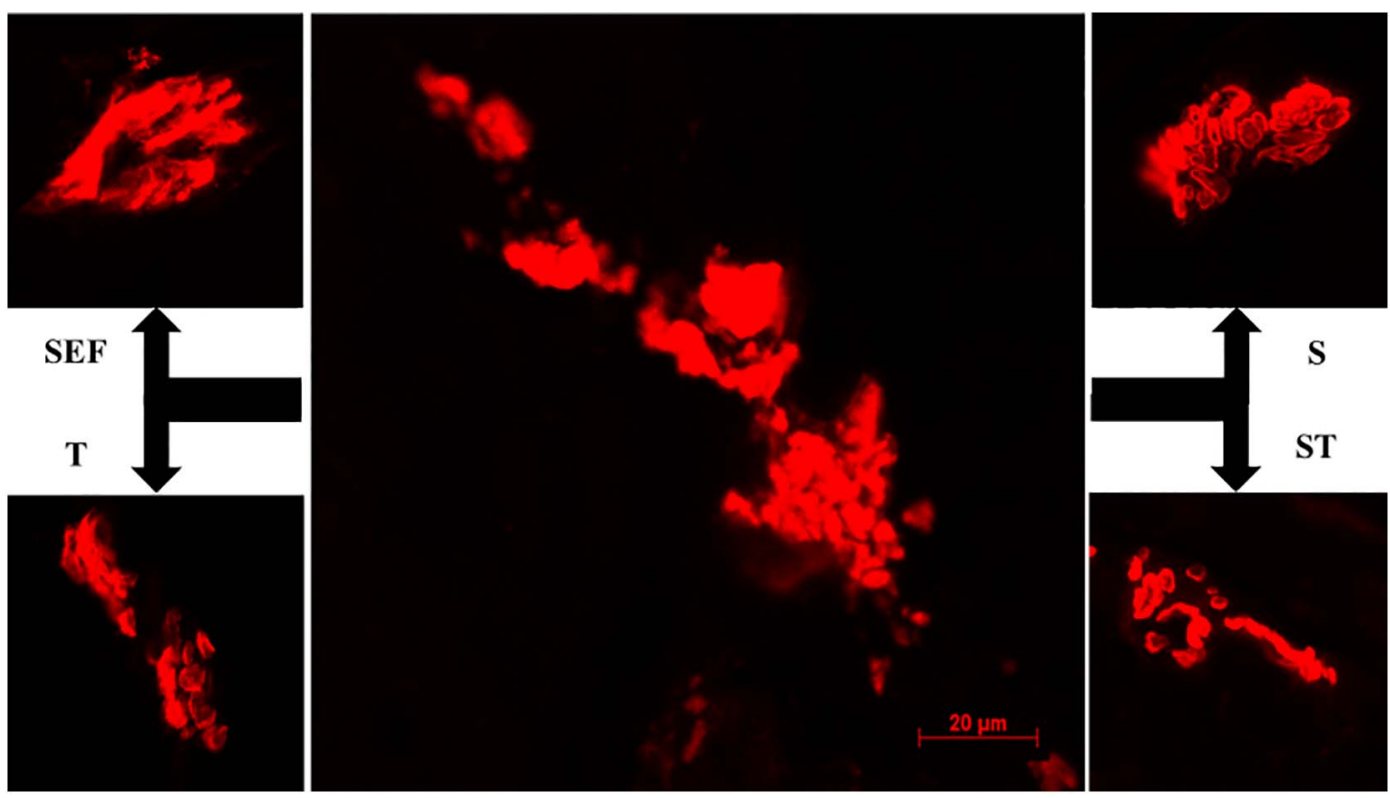

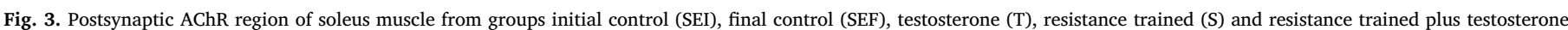
(ST). Stained with rhodamine $\alpha$-bungarotoxin. $1000 \times$ magnification.

$(p<0.05)$. There was no statistical difference for any other variable.

\subsection{Skeletal muscles}

\subsubsection{Soleus}

Fig. 5 presents comparative images of all groups. T, S and ST groups showed the same magnitude of type I myofibers hypertrophy, thus statistically different from SEI and SEF $(p<0.05)$. ST group presented cross-sectional area of the type IIa myofibers larger than SEF $(p<0.05)$. The volume density of type I myofibers was shown to be lower in ST than SEI $(p<0.05)$. As for type IIa myofibers, ST increased Vv [type IIa] compared to SEI and SEF $(p<0.05)$. Data are presented in Table 4.

\subsubsection{Plantaris}

$\mathrm{T}$ group significantly hypertrophied type I myofibers compared to SEI and SEF $(p<0.05)$. Groups S and ST demonstrated significant increases of type I myofibers compared to SEI and SEF ( $p<0.05)$. As for type IIx myofibers, both groups showed to be larger than SEI $(p<0.05)$. However, only ST group had significant enlargement compared to SEF $(p<0.05)$. Data are presented in Table 5. Fig. 6 presents comparative images of all groups.

\section{Discussion}

It is known that anthropometric parameters may vary during the aging process. This is mainly due to the metabolic and morphological changes characteristic of this period. In the present study, we observed a significantly difference in the baseline body weight of the SEF, S and ST groups compared to the other groups. This fact did not in any way influenced the results, since the comparisons were made in predetermined periods, taking into account these probable variations (data presented in Table 1). Thus, the fact that called us more attention was that the groups that represented the aging process (SEI vs SEF) did not show statistical difference at the end of the experiment. This outcome is variable in the literature and may be related to the age, lineage and rodent investigated.

At the end of the experiment, the ST group had body mass significantly lower than SEF and S. This fact can be explained by the reduction of body fat mass induced by the sum of the use of anabolic steroids and resistance training, already widely described in the literature (Krause Neto et al., 2015; Lambert et al., 2002). However, since we did not evaluate the body composition of the groups in question, such an assertion is based exclusively on evidence. Clearly, homeostatic changes can lead to more lasting changes over total body weight.

Table 3

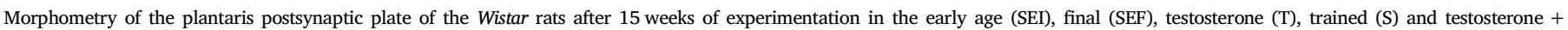
trained (ST).

\begin{tabular}{|c|c|c|c|c|c|c|c|}
\hline \multirow[b]{2}{*}{ Variables } & \multicolumn{5}{|l|}{ Groups } & \multirow[b]{2}{*}{$\mathrm{F}$} & \multirow[b]{2}{*}{$p$ value } \\
\hline & SEI & SEF & $\mathrm{T}$ & $S$ & ST & & \\
\hline \multicolumn{8}{|l|}{ Non-stained receptors } \\
\hline Total perimeter $(\mu \mathrm{m})$ & $95,66 \pm 20$ & $126,48 \pm 26,98$ & $155,02 \pm 26,54^{\mathrm{a}}$ & $109,47 \pm 29,75$ & $124,15 \pm 23,99$ & 5698 & 0,001 \\
\hline Total area $\left(\mu \mathrm{m}^{2}\right)$ & $615,24 \pm 239,49$ & $1115,85 \pm 456,52$ & $1544,65 \pm 524,18^{\mathrm{a}}$ & $845,57 \pm 427,61$ & $1092,33 \pm 374,54$ & 5237 & 0,002 \\
\hline \multicolumn{8}{|l|}{ Stained receptors } \\
\hline Perimeter $(\mu \mathrm{m})$ & $140,72 \pm 41,87$ & $197,48 \pm 66,75$ & $243,1 \pm 85,1$ & $151 \pm 59,06$ & $174,88 \pm 43,71$ & 3615 & 0,112 \\
\hline Area $\left(\mu \mathrm{m}^{2}\right)$ & $401,01 \pm 201,3$ & $673,66 \pm 253,61$ & $967,04 \pm 340,42^{\mathrm{a}}$ & $493,29 \pm 206,83$ & $729,85 \pm 252,12$ & 5062 & 0,002 \\
\hline Dispersion (\%) & $63,32 \pm 10,28$ & $62,03 \pm 8,52$ & $62,88 \pm 9,18$ & $62,01 \pm 10,82$ & $67,51 \pm 10,06$ & 0,252 & 0,935 \\
\hline
\end{tabular}

Values are presented as mean \pm standard deviation.

Dispersion $=$ stained area/total non-stained area $\times 100$.

a indicates significant difference relative to SEI $(p \leq 0.05)$. 


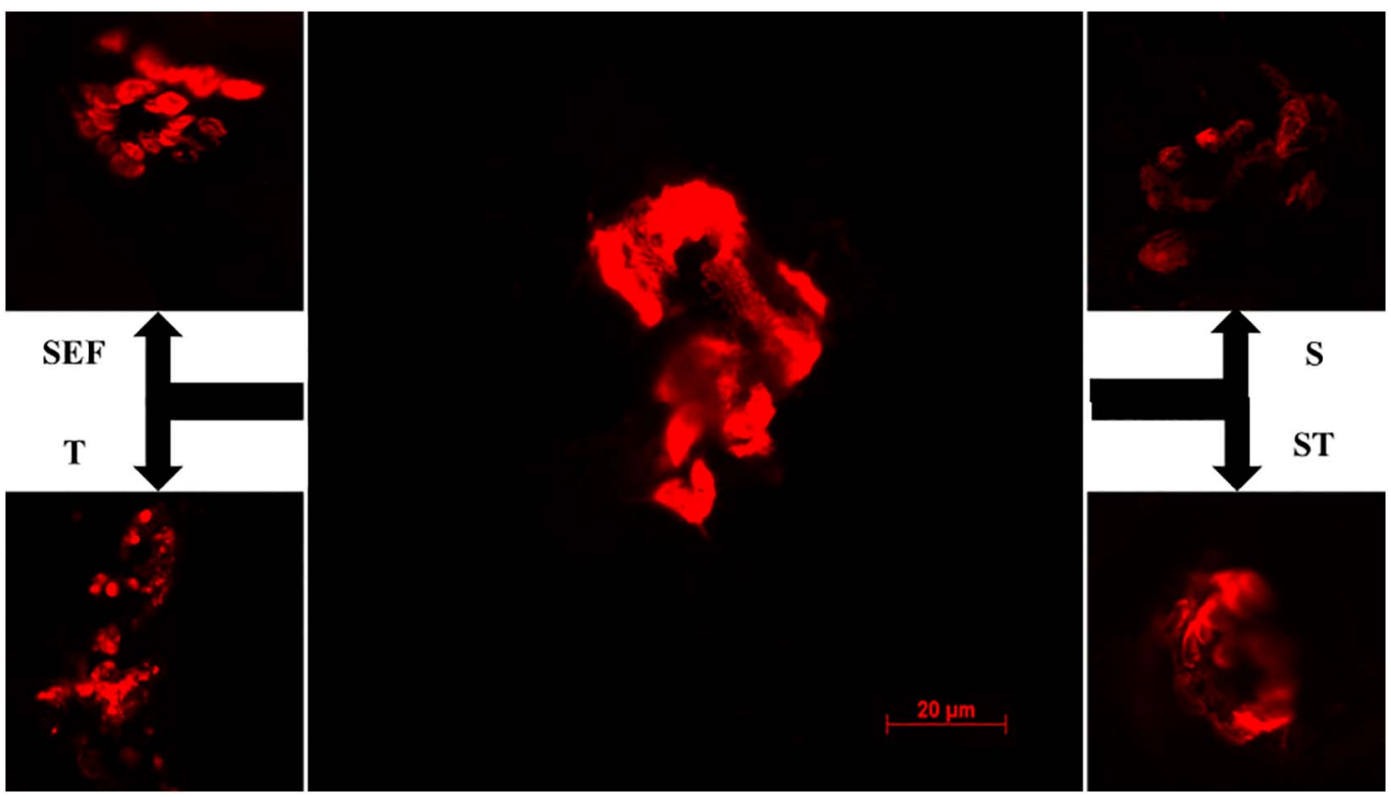

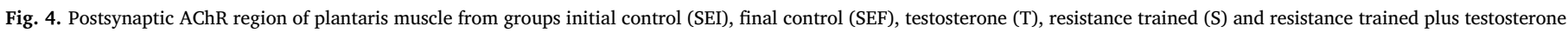
(ST). Image stained with rhodamine $\alpha$-bungarotoxin. $1000 \times$ magnification.

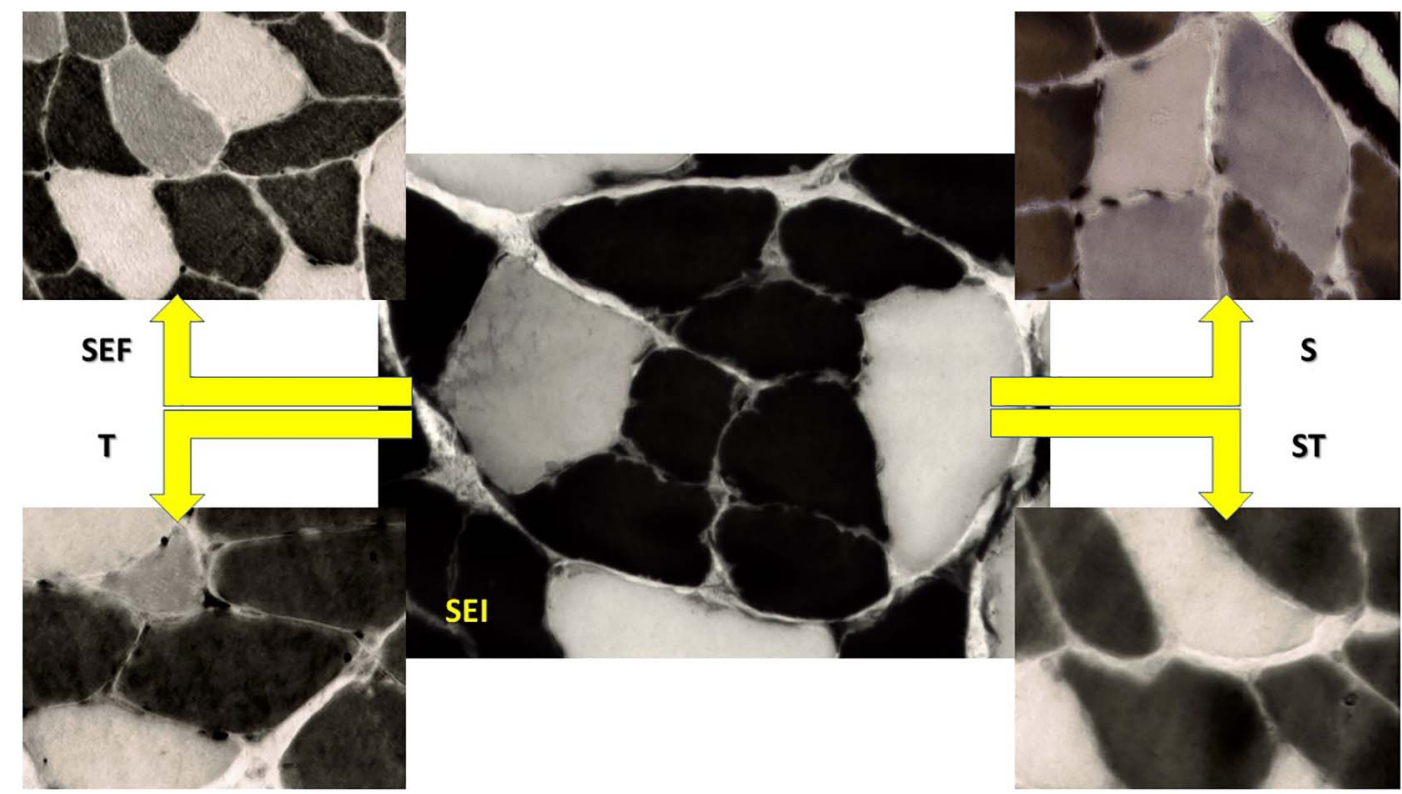

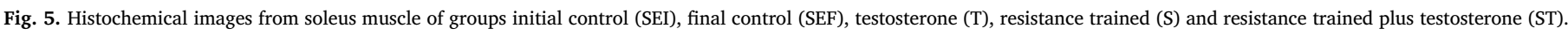
Image stained with ATPase. $400 \times$ magnification.

Analyzing the pre-to post-intervention moments, we identified that T, S, and ST had a significant body weight decrease. The reductions were equivalent to $14.7,9.1$ and $14.9 \%$, respectively. Knowing that the energetic expenditure induced by the subsequent sessions of resistance exercise, causes the metabolism to oxidize a higher percentage of body fat and stimulate a continuous increase of muscle mass, it was expected that the $S$ group had a significant reduction of this parameter. However, by associating resistance training with testosterone, body weight reduction was even more significant. Interestingly, the use of isolated testosterone propionate was as potent as the other interventions in reducing body weight.

The weight of SL and PL muscles, together with their respective ratios, presented different responses at the end of the study. The SL muscle was not influenced by either the aging process or the administered interventions. The PL ratio decreased $14.8 \%$ compared to SEI. Our data are in part similar to others found in the literature. Deschenes et al. (Deschenes et al., 2010) demonstrated that both SL and PL weights did not change between 10 and 21 months of age. This was corroborated by Deschenes et al. (Deschenes et al., 2015) that presented similar results. However, the ratio of both muscles decreased significantly in these respective ages (Deschenes et al., 2010). Again, it is worth emphasizing that the different ages and lineage of the animals may explain possible differences found in the results.

Comparing the results of the interventions, we found that $\mathrm{T}, \mathrm{S}$ and ST showed higher ratios than SEF. It is known that both resistance training and exogenous testosterone can stimulate increased muscle size, or even prevent the sarcopenic effect characteristic of aging. Thus, both interventions seem to have been sufficient to avoid the reduction of muscle mass, for which was demonstrated in the analysis of the PL muscle ratio. 
Table 4

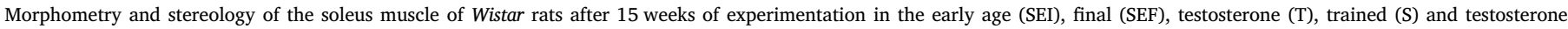
+ trained (ST) groups.

\begin{tabular}{|c|c|c|c|c|c|c|c|}
\hline \multirow[b]{2}{*}{ Variables } & \multicolumn{5}{|l|}{ Groups } & \multirow[b]{2}{*}{$\mathrm{F}$} & \multirow[b]{2}{*}{$p$ value } \\
\hline & SEI & SEF & $\mathrm{T}$ & $S$ & ST & & \\
\hline \multicolumn{8}{|c|}{ Cross-sectional area $\left(\mu \mathrm{m}^{2}\right)$} \\
\hline Type I & $2851,71 \pm 696,45$ & $3067,56 \pm 745,62$ & $3665,05 \pm 973,12^{\mathrm{a}, \mathrm{b}}$ & $3698,31 \pm 912,95^{\mathrm{a}, \mathrm{b}}$ & $3896,53 \pm 1014,58^{\mathrm{a}, \mathrm{b}}$ & 12,913 & 0,000 \\
\hline Type IIa & $3481,17 \pm 963,97$ & $3326,74 \pm 828,09$ & $3704,17 \pm 1045,3$ & $3665,67 \pm 1214,03$ & $4247,68 \pm 711,66^{b}$ & 3,321 & 0,008 \\
\hline $\begin{array}{l}\text { Type IIx } \\
\text { Vv }\end{array}$ & $3276,21 \pm 737,22$ & $3217,15 \pm 1741,21$ & $3881,9 \pm 1237,92$ & $3777,11 \pm 1019,59$ & $3999,57 \pm 402,23$ & 0,688 & 0,636 \\
\hline Type I & $87,02 \pm 2,88$ & $86,6 \pm 2,68$ & $84,24 \pm 2,95$ & $83,91 \pm 2,9$ & $81,53 \pm 4,08^{\mathrm{a}}$ & 4,450 & 0,002 \\
\hline Type IIa & $6,07 \pm 1,29$ & $6,63 \pm 1,31$ & $10,44 \pm 2,86$ & $10,49 \pm 1,95$ & $13,36 \pm 3,53^{\mathrm{a}, \mathrm{b}}$ & 8,100 & 0,000 \\
\hline Type IIx & $3,89 \pm 2,58$ & $3,55 \pm 2,52$ & $2,33 \pm 1,23$ & $2,64 \pm 0,72$ & $2,55 \pm 1,02$ & 1,355 & 0,256 \\
\hline Intersticium & $2,98 \pm 0,99$ & $3,18 \pm 1,02$ & $2,96 \pm 0,61$ & $2,92 \pm 1,41$ & $2,52 \pm 0,78$ & 1,768 & 0,135 \\
\hline
\end{tabular}

Values are presented as mean \pm standard deviation.

$\mathrm{Vv}=$ volume density (\%).

${ }^{a}$ indicates a significant difference in relation to SEI $(p \leq 0.05)$.

${ }^{\mathrm{b}}$ indicates significant difference in relation to SEF $(p \leq 0.05)$.

According to literature, it is evident that there is an increase in the motor end-plate region (total area) during aging. Li et al. (Li et al., 2011) explained that the dispersion of the end-plate is a result of its fragmentation, caused by a loss of balance between degeneration and regeneration processes. According to Deschenes et al. (Deschenes et al., 2010), the fragmentation of NMJ precedes the onset of sarcopenia. Being so, there are several mechanisms that may be involved in this regulatory loss. Ibebunjo et al. (Ibebunjo et al., 2012) analyzed the genomic profile of rat muscles and demonstrated a reduction of mitochondrial function, directing these tissues to sarcopenia. A balance should also be maintained between the Agrin (training) and neurotropsin (degeneration) proteins in the postsynaptic structure of the NMJ. Drey et al. (Drey et al., 2013) suggest that the C-terminal fragment of agrin may be a potential marker for sarcopenia caused by degeneration of NMJ. Hettwer et al. (Hettwer et al., 2013) corroborate the previous study, demonstrating that sarcopenia is an agrina-dependent process. Other mechanisms, such as reduction of tyrosine kinase receptor (TrkB), myogenic regulation factor 4 (MRF-4), IGF-1 and ROS enhancement may also be involved in NMJ fragmentation (Krause Neto and Gama, 2013). Despite all of these data, our study did not identify any significant changes in the postsynaptic component. This can be explained by the fact that our study started from an age (20 monthsold) whose alterations could already be present and a longer time of analysis was necessary (age further than 24 months-old).

The use of TP did not change any parameters investigated in the soleus muscle. However, the plantaris showed a significant difference of $62.05 \%$ in the total perimeter compared to SEI. Also, total area of ACh receptors in the $\mathrm{T}$ group was statistically higher than SEI. As for the stained receptors, area was $141.15 \%$ larger in T than SEI. The dispersion of stained receptors was not altered by the use of TP. Also, we did not find any statistical difference between $\mathrm{T}$ and SEF groups. Nudler et al. (Nudler et al., 2005) demonstrated that testosterone can regulate the mechanisms of coupling between calcium channels and transmission in the NMJ of sexually dysmorphic motoneurons. Bleisch and Harrelson (Bleisch and Harrelson, 1989) have demonstrated that androgens modulate motor terminal plate size and AChRs density in the synapse of the levator ani muscle. Monks et al. (Monks et al., 2004) cite that the expression of ARs in the region of the terminal end-plate may be different between some types of muscles and mainly depending on the type of muscle fibers within these muscles. Studies with other animal species suggest that testosterone may potentiate the differences in androgen responsiveness of motor units [MUs] (Nagaya and Herrera, 1995). In this case, MUs with slow-twitch myofibers are less responsive than those with faster myofibers. This would explain our data, where only the end-plate of the plantar muscle was adjusted in the T group. Blanco et al. (Blanco et al., 2001) investigated the effect of 28-day TP administration on intact male rats. The authors verified an increase of $81.7 \%$ of ARs in the motor neurons of the treated group. These data make clear the range of action of testosterone in various PNS structures. However, we must make clear the absence of studies on such aged model facts.

The aging process can clearly influence the adaptations in NMJ promoted by endurance training (Deschenes et al., 2011). However, it appears that resistance training may have different effects. In the study

Table 5

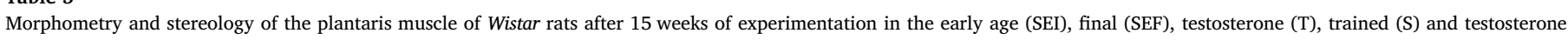
+ trained (ST).

\begin{tabular}{|c|c|c|c|c|c|c|c|}
\hline \multirow[b]{2}{*}{ Variables } & \multicolumn{5}{|l|}{ Groups } & \multirow[b]{2}{*}{$\mathrm{F}$} & \multirow[b]{2}{*}{$p$ value } \\
\hline & SEI & SEF & $\mathrm{T}$ & $\mathrm{S}$ & ST & & \\
\hline \multicolumn{8}{|c|}{ Cross-sectional area $\left(\mu \mathrm{m}^{2}\right)$} \\
\hline Type I & $2023,24 \pm 631,99$ & $2179,36 \pm 443,25$ & $2647,83 \pm 526,95^{\mathrm{a}}$ & $2880,23 \pm 475,14^{\mathrm{a}, \mathrm{b}}$ & $2974,55 \pm 712,23^{\mathrm{a}, \mathrm{b}}$ & 8509 & 0,000 \\
\hline Type IIa & $3442,3 \pm 1029,06$ & $3391,02 \pm 815,49$ & $3556,34 \pm 977,46$ & $3905,25 \pm 1288,9$ & $4015,44 \pm 1586,6$ & 2567 & 0,027 \\
\hline $\begin{array}{l}\text { Type IIx } \\
\text { Vv }\end{array}$ & $2579,18 \pm 663,36$ & $2686,24 \pm 753,94$ & $2825,2 \pm 455,1$ & $3084,54 \pm 689,53^{a}$ & $3341,44 \pm 503,84^{\mathrm{a}, \mathrm{b}}$ & 8561 & 0,000 \\
\hline Type I & $10,06 \pm 2,56$ & $10,56 \pm 3,58$ & $7,32 \pm 5,95$ & $6,56 \pm 3,49$ & $6,5 \pm 4,34$ & 2054 & 0,086 \\
\hline Type IIa & $71,62 \pm 3,21$ & $71,54 \pm 8,35$ & $76,49 \pm 11,65$ & $77,13 \pm 6,04$ & $77,36 \pm 11,59$ & 1237 & 0,305 \\
\hline Type IIx & $15,54 \pm 3,96$ & $14,79 \pm 7,54$ & $13,82 \pm 10,28$ & $14,33 \pm 3,84$ & $13,92 \pm 8,85$ & 0,225 & 0,950 \\
\hline Intersticium & $2,74 \pm 1,13$ & $3,08 \pm 1,67$ & $2,2 \pm 0,49$ & $1,94 \pm 0,72$ & $2,05 \pm 0,8$ & 2683 & 0,031 \\
\hline
\end{tabular}

Values are presented as mean \pm standard deviation.

$\mathrm{Vv}=$ volume density (\%).

${ }^{\mathrm{a}}$ indicates significant difference from SEI $(p \leq 0.05)$.

${ }^{\mathrm{b}}$ indicates significant difference from SEF $(p \leq 0.05)$. 


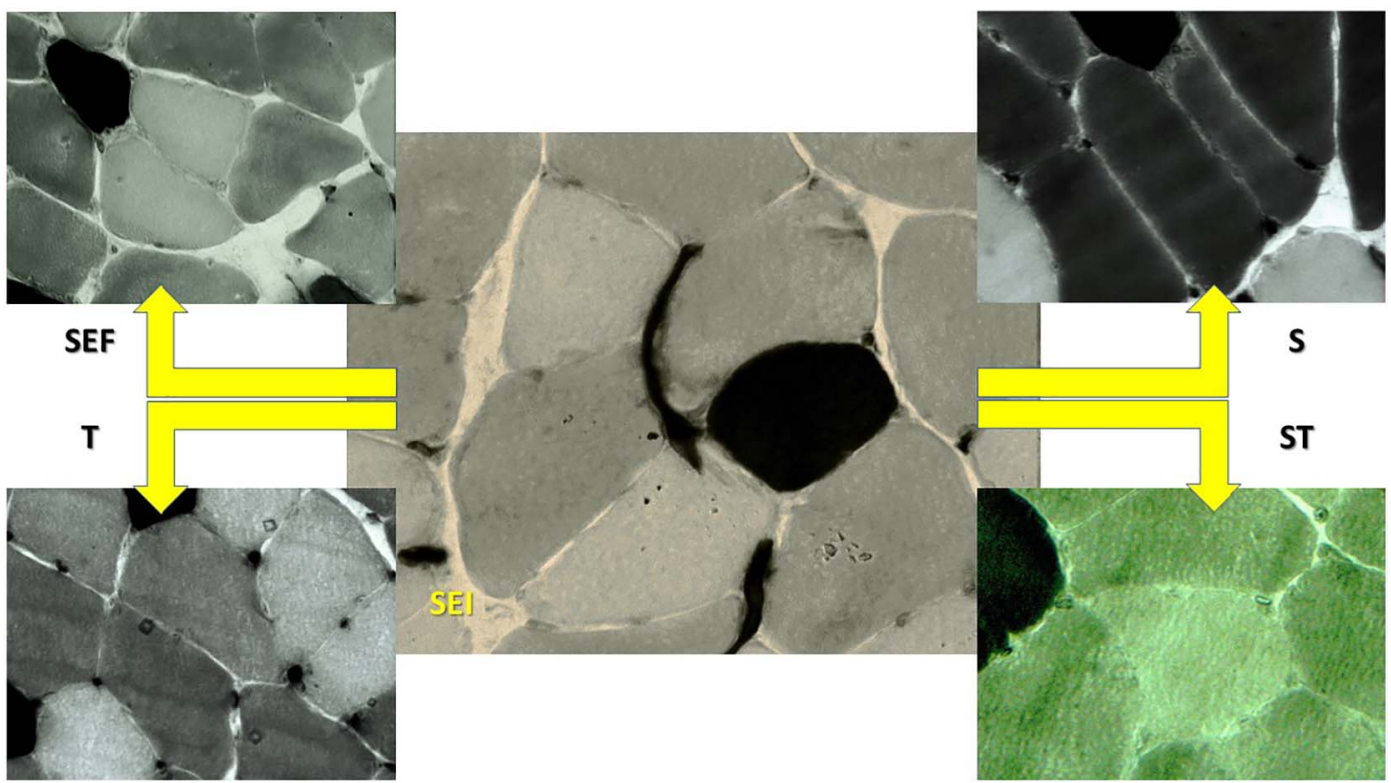

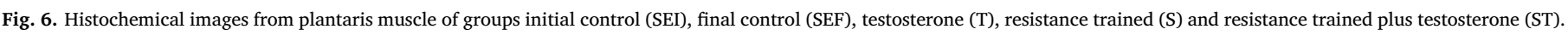
Image stained with ATPase. $400 \times$ magnification.

by Deschenes et al. (Deschenes et al., 2015), there was a significant increase induced by RT in only the stained and total areas of the muscle fibers of fast contraction of the soleus muscle. As for the NMJ of the plantaris muscle, the data presented by the authors were similar to ours. Deschenes et al. (Deschenes et al., 2015) explain that the type II muscle fibers of the soleus are less recruited than those of type I, making them more sensitive to the training stimulus.

Interestingly, it was demonstrated by the association between RT and TP. Both areas of the stained and total end-plates of the soleus muscle were statistically smaller in ST than SEF. However, in the plantar muscle, there was no statistical difference between the parameters measured. Possibly, future studies should address this issue.

Verdijk et al. (Verdijk et al., 2007) demonstrated that type II myofibers of the vastus lateralis of the elderly present reduced amount of satellite cells when compared to young. The authors were the first to demonstrate a relationship between muscle atrophy of type II myofibers of older muscles with decline in satellite cell content in fiber-specific type. Further, Desaki (Desaki, 2008) mentioned that there is a difference between structural changes in muscle fibers in aging, and it may be a reduction in the number of muscle fibers or in their size. Tauchi et al. (Tauchi et al., 1971) observed structural changes in the muscles of Wistar rats during aging, which glycolytic fibers decreased in size, but not in number, while oxidative fibers decreased in number but not in size. In our study, we demonstrated that there was no significant change in morphometry and stereology of type I, IIa or IIx myofibers in the soleus and plantaris during aging. Our results are corroborated by Deschenes et al. (Deschenes et al., 2010), however, Deschenes et al. (Deschenes et al., 2011) demonstrated significant atrophy of type I myofibers of the soleus in the age group. In addition, plantar muscle morphometry indicated a significant reduction of type IIa myofibers and an increase in the total proportion of type I myofibers. Deschenes et al. (Deschenes et al., 2012) found a significant reduction of all types of myofibers, with an increase in the proportion of type I in the soleus. Deschenes et al. (Deschenes et al., 2013b) reported a decrease in the cross-sectional area of type II myofibers, with an increase in the proportion of type I fibers and type II reduction in the soleus muscle. In addition, the plantaris muscle had an increase in the area of fibers I and IIa, with reduction of IIx fibers. In 2015, Deschenes et al. found a significant increase in the proportion of type I fibers and plantaris muscle type II enlargement, without any change in the cross-sectional area of these fibers (Deschenes et al., 2015). Finally, Deschenes et al.
(Deschenes et al., 2016) demonstrated a reduction in the area of the type I and IIa fibers, with an increase in the proportion and occupied area of the plantaris muscle type IIx fibers. The divergence in the presented results may have been influenced by the variation of the lineages and different ages of the rodents investigated.

Clearly, testosterone treatment increases the muscle mass of aged individuals (Kovacheva et al., 2010). Our results demonstrated that in the soleus muscle the CSA of type I muscle fibers was $28.5 \%$ and $19.5 \%$ higher than SEI and SEF, respectively. Already in the plantaris muscle, the CSA of the muscle fibers of the T group presented $30.9 \%$ higher than SEI. However, other parameters did not change significantly. Other studies have also observed muscle hypertrophy in young and old, submitted to the use of testosterone, and in these studies were also demonstrated increase in the number of myonuclei and satellite cells (Sinha-Hikim et al., 2002; Sinha-Hikim et al., 2006). Many mechanisms could be responsible for muscle hypertrophy such as inactivation of the enzyme JNK (stress-activated enzymes) with activation of p38 MAPK (enzyme capable of stimulating muscle growth), activating Notch signaling (Brown et al., 2009). As known, Notch signaling is essential for myogenic progression of the satellite cells required for muscle regeneration and repair processes. Kovacheva et al. (Kovacheva et al., 2010) found that both fast and slow-twitch muscle fibers are significantly increased by testosterone treatment through suppression of myostatin and JNK, thus stimulating muscle gains in the aged animals. Kawada et al. (Kawada et al., 2006) also verified significant reductions in myostatin content in the gastrocnemius and soleus muscles. However, it seems that the hypertrophic response to anabolic steroid use alone is dose-dependent (Woodhouse et al., 2003). According to Katznelson et al. (Katznelson et al., 2006) therapeutic dosages of anabolic steroids may not be sufficient for muscular hypertrophy, therefore, supraphysiological doses may have a greater effect.

Resistance training has been considered the most effective intervention in decreasing rates of muscle loss, and maintaining or increasing the muscular strength of the aged (Lynch et al., 2007). RT effects induced by the subsequent sessions produce a cumulative signaling, eventually leading to changes in the muscular CSA. In the soleus of trained animals, CSA of type I muscle fibers was $29.7 \%$ and $20.5 \%$ larger than SEI and SEF. In plantaris, type I muscle fibers increased significantly compared to SEI and SEF. The CSA of type IIx fibers increased significantly in comparison to SEI. Interestingly, RT is as effective as testosterone in increasing the expression of mRNA for growth 
factors that stimulate aggregation of new proteins (Snijders et al., 2009). According to Carey et al. (Carey et al., 2007), the loss imposed by the aging process to the Notch complex can be modulated by resistance training. Olsen et al. (Olsen et al., 2006) demonstrated that increases in muscle cell size were concomitant to satellite cell content increases imposed by resistance training. In addition, other studies were able to demonstrate a similar response in elderly individuals (Mackey et al., 2007; Verney et al., 2008). Using a 21-week protocol, Ahtiainen et al. (Ahtiainen et al., 2011) were able to demonstrate muscle hypertrophy of in both young and aged groups.

Studies using the LC model have demonstrated divergent results in muscle hypertrophy. Yarasheski, Lemon and Gilloteaux (Yarasheski et al., 1990) demonstrated that vertical climbing models were adequate to stimulate muscular hypertrophy. However, Duncan, Williams and Lynch (Duncan et al., 1998) failed to show this same phenomenon. The comparison between the two works is difficult due to different protocols and evaluation of the skeletal muscles. In addition, Hornberger and Farrar (Hornberger and Farrar, 2004) described the climbing equipment and training protocol currently used, with positive effects on flexor hallux longus muscle hypertrophy (FHL). Lee et al. (Lee et al., 2004) also showed that climbing training increased muscle mass of the FHL muscle in young adult rats. This effect was potentiated when combined with overexpression of IGF-1. Corroborating, Kraemer et al. (Kraemer et al., 2013) showed that resistance training induced dynamic adaptations in somatotrophic structure and function. These facts may explain the effect of IGF on the stimulus of muscle hypertrophy, since growth hormone (GH) and IGF-1 work together. Intriguingly, Lee et al. (Lee et al., 2004) reported that LC equipment is modest in intensity when compared to other devices that used a greater number of repetitions and less frequency of training sessions. Hellyer et al. (Hellyer et al., 2012) demonstrated that even moderate, LC was sufficient to increase AST and MHC content in the FHL muscle of young adolescent rats. However, this study demonstrated that even stimulating muscle hypertrophy, this type of training failed to increase expression and phosphorylation of key factors (Akt/mTOR and rpS6) for muscle hypertrophy. The authors suggest that the mechanisms that stimulate muscle hypertrophy should be different between young and adult rats. In addition, other important critical factors may be related to skeletal muscle hypertrophy. Begue et al. (Begue et al., 2013) investigated whether high-intensity training could regulate IL-6/STAT1/STAT3 activity. Recent studies have shown that this cell signaling pathway is important for muscle hypertrophy (Trenerry et al., 2007; Trenerry et al., 2011). The authors compared the response of these molecules after 2, 4 and 10 weeks of vertical climbing training, with significant gains in strength and muscle hypertrophy after ten weeks of training. In addition, the acute measurements showed increased expression of IL-6 mRNA concomitant with phosphorylation of STAT1 and STAT3 after 2 and $6 \mathrm{~h}$ of training. A positive BrdU analysis demonstrated increased satellite cells after training. Corroborating, Luo et al. (Luo et al., 2013) demonstrated that RT was effective in enhancing autophagy and reducing apoptosis of muscle cells by modulating IGF-1 and its receptors, in addition to the activation of Akt/mTOR and Akt/FOXO3 in the muscle of old rats. Although this evidence has demonstrated the activation of relevant cellular and molecular pathways that explain muscle hypertrophy observed in studies using the LC model, further studies with rats at different stages of senescence are needed.

Increased fat-free mass gain can be achieved by using resistance training and testosterone administration (Bhasin et al., 2001). The ST group presented CSA of SL type I muscle fiber greater than SEI and SEF. The CSA of type IIa muscle fibers were larger than SEF. The density of muscle fibers of type IIa was higher than SEI and SEF. As for the plantaris muscle, the ST group presented AST of type I fibers $47.02 \%$ and $36.5 \%$ significantly higher than SEI and SEF, respectively. Regarding the area of type IIx fibers, the difference was $29.5 \%$ and $24.4 \%$ in relation to SEI and SEF. These results suggest that increasing the fatfree mass makes it possible to increase or maintain the body weight of aged individuals, as well as to reduce body fat gain (Isidori et al., 2005; Peterson et al., 2010). Lambert et al. (Lambert et al., 2002) agreed that the combination of RT and exogenous testosterone leads to greater muscular gains. In agreement, Bhasin et al. (Bhasin et al., 1996; Bhasin et al., 2000) demonstrated that the association of resistance training and anabolic steroid is more effective in increasing muscle mass and strength than hormone treatment or resistance training alone. Interestingly, our data demonstrate that both the combination of RT and TP, as well as each single therapy, were not different from each other. It is known that testosterone acts via interaction with its respective receptor (AR), so resistance training is able to increase AR mRNA expression (Willoughby and Taylor, 2004), enhancing the effects of its administration.

Both groups submitted to resistance training presented similar results for muscular hypertrophy and increase of the maximum carrying load and the results can be explained by the fact that the total load carried is proportional between the two groups at the end of the fifteen weeks of training. This fact suggests that laboratory animals may have the adaptive response to training load in a manner similar to that found in humans.

In conclusion, our findings suggest that both therapies alone or together have little effect on the morphology of the postsynaptic region of distinct muscles. Moreover, the three therapies are potentially stimulating for strength gains and muscle hypertrophy. Finally, the equalization of the absolute training load volume seems to exert similar effects between rodents and those seen in humans. Future researchers should investigate other parameters of NMJ such as AChRs density, basal lamina invagination depth and synaptic cleft enlargement.

\section{Conflict of interest}

All authors disclose any conflicts of interest.

\section{Acknowledgment}

We would like to thank the help given by Professor Michael Deschenes from the College of William \& Mary. We thank the access to equipment and assistance provided by the National Institute of Science and Technology on Photonics Applied to Cell Biology (INFABIC) at the State University of Campinas; INFABIC is co-funded by Fundação de Amparo a Pesquisa do Estado de São Paulo (FAPESP) (08/57906-3) and Conselho Nacional de Desenvolvimento Cientifico e Tecnológico (CNPq) (573913/2008-0). Also, we would like to thank the technicians of the laboratory of morphology and physical activity of Universidade Estadual Paulista (UNESP-Rio Claro).

\section{Author contributions}

WKN trained the rodents, participated in euthanasia and collected the material, prepared and analyzed the laminas and wrote the final version of the manuscript. WAS trained the rodents, participated in euthanasia and collected the material and final discussion of the results. APC participated in the collection of tissues, purchase of material, evaluation of results and final discussion of the manuscript. CAA participated in the collection of tissues, purchase of material, evaluation of results and final preparation of the manuscript. EFG guided all stages of this work, being of fundamental importance for the presentation of these results.

\section{References}

Ahtiainen, J.P., Hulmi, J.J., Kraemer, W.J., Lehti, M., Nyman, et al., 2011. Heavy resistance exercise training and skeletal muscle androgen receptor expression in younger and older men. Steroids 76, 183-192.

Begue, G., Doullard, A., Galbes, O., Rossano, B., Vernus, B., Candau, R., Py, G., 2013. Early activation of rat skeletal muscle IL-6/STAT1/STAT3 dependent gene expression in resistance exercise linked to hypertrophy. PLoS One 8 (2), e57141. 
Bhasin, S., Storer, T.W., Berman, N., Callegari, C., et al., 1996. The effect of supraphysiologic doses of testosterone on muscle size and strength in normal men. N. Engl. J. Med. 335 (1), 1-7.

Bhasin, S., Storer, T.W., Javanbakht, M., Berman, N., Yarasheski, K.E., Phillips, J., Dike, M., Sinha-Hikim, I., Shen, R., Hays, R.D., Beall, G., 2000. Testosterone replacement and resistance exercise in HIV-infected men with weight loss and low testosterone levels. JAMA 283, 763-770.

Bhasin, S., Woodhouse, L., Storer, T.W., 2001. Proof of the effect of testosterone on skeletal muscle. J. Endocrinol. 127, 27-38.

Blanco, C.E., Davenport, T., Wachi, S., Goedken, T., 2001. Androgen receptor immunoreactivity of male rat cervical motor neurons is increased by chronic pharmacologic testosterone treatment. Acta Physiol. Pharmacol. Bulg. 26 (1-2), 7-10.

Bleisch, W.V., Harrelson, A., 1989. Androgens modulate end-plate size and ACh receptor density at synapses in rat levator ani muscle. J. Neurobiol. 20 (4), 189-202.

Brown, D., Hikim, A.P., Kovacheva, E.L., Sinha-Hikim, I., 2009. Mouse model of testosterone-induced muscle fiber hypertrophy: involvement of p38 mitogen-activated protein kinase-mediated Notch signaling. J. Endocrinol. 201 (1), 129-139.

Cappello, V., Vezzoli, E., Righi, M., Fossati, M., Mariotti, R., Crespi, A., ... Francolini, M., 2012. Analysis of neuromuscular junctions and effects of anabolic steroid administration in the SOD1G93A mouse model of ALS. Mol. Cell. Neurosci. 51 (1), 12-21.

Carey, K.A., Farnfield, M.M., Tarquinio, S.D., Cameron-Smith, D., 2007. Impaired expression of Notch signaling genes in aged human skeletal muscle. J. Gerontol. Biol. Sci. 62, 9-17.

Carson, J.A., Lee, W.J., McClung, J., Hand, G.A., 2002. Steroid receptor concentration in aged rat hindlimb muscle: effect of anabolic steroid administration. J. Appl. Physiol. 93, 242-250.

Cavalcante, W.L.G., Dal Pai-Silva, M., Gallacci, M., 2004. Effects of nandrolone decanoate on the neuromuscular junction of rats submitted to swimming. Comp. Biochem. Physiol. C Toxicol. Pharmacol. 139 (4), 219-224.

Desaki, J., 2008. Regeneration of muscle fibers in the extensor digitorium longus muscle of the aged rat. J. Electron Microsc. 57 (2), 59-66.

Deschenes, M.R., Judelson, D.A., Kraemer, W.J., Meskaitis, V.J., Volek, J.S., Nindl, B.C., Harman, F.S., Deaver, D.R., 2000. Effects of resistance training on neuromuscular junction morphology. Muscle Nerve 23, 1576-1581.

Deschenes, M.R., Roby, M.A., Eason, M.K., Harris, M.B., 2010. Remodeling of the neuromuscular junction precedes sarcopenia related alterations in myofibers. J. Physiol. 45, 389-393.

Deschenes, M.R., Roby, M.A., Glass, E.K., 2011. Aging influences adaptations of the neuromuscular junction to endurance training. Neuroscience 190, 56-66.

Deschenes, M.R., Sherman, E.G., Glass, E.K., 2012. The effects of pre-habilitative conditioning on unloading-induced adaptations in young and aged neuromuscular systems. Exp. Gerontol. 47, 687-694.

Deschenes, M.R., Hurst, T.E., Ramser, A.E., Sherman, E.G., 2013a. Presynaptic to postsynaptic relationship of the neuromuscular junction are held constant across age and muscle fiber type. Dev. Neurobiol. 73 (10), 744-753.

Deschenes, M.R., Gaertner, J.R., O'Reilly, S., 2013b. The effects of sarcopenia on muscles with different recruitment patterns and myofiber profiles. Curr. Aging Sci. 6 (3), 266-272.

Deschenes, M.R., Sherman, E.G., Roby, M.A., Glass, E.K., Harris, M.B., 2015. Effect of resistance training on neuromuscular junctions of young and aged muscles featuring different recruitment patterns. J. Neurosci. Res. 93 (3), 504-513.

Deschenes, M.R., Kressin, K.A., Garratt, R.N., Leathrum, C.M., Shaffrey, E.C., 2016. Effects of exercise training on neuromuscular junction morphology and pre- to postsynaptic coupling in young and aged rats. Neuroscience 1 (316), 167-177.

Dias, R.M.R., Cyrino, E.S., Salvador, E.P., Caldeira, L.F.S., Nakamura, F.Y., Papst, R.R., Gurjão, A.L.D., 2005. Influência do processo de familiarização para avaliação da força muscular em testes de 1-RM. Rev. Bras. Med. Esporte 11 (1), 34-38.

Drey, M., Sieber, C.C., Bauer, J.M., Uter, W., Dahinden, P., Fariello, R.C., Vrijbloed, J.W., 2013. C-terminal Agrin fragment as a potential marker for sarcopenia caused by degeneration of the neuromuscular junction. Exp. Gerontol. 48, 76-80.

Duncan, N.D., Williams, D.A., Lynch, G.S., 1998. Adaptations in rat skeletal muscle fol lowing long-term resistance exercise training. Eur. J. Appl. Physiol. 77, 372-378.

Fitts, J.M., Klein, R.M., Powers, C.A., 2004. Comparison of tamoxifen and testosterone propionate in male rats: differential prevention of orchidectomy effects on sex organs, bone mass, growth, and the growth hormone-IGF-I axis. J. Androl. 25 (4), 523-534.

Harris, M.B., Slack, K.N., Prestosa, D.T., Hryvniak, D.J., 2010. Resistance training improves femoral artery endothelial dysfunction in aged rats. Eur. J. Appl. Physiol. 108, 533-540.

Hellyer, N.J., Nokleby, J.J., Thicke, B.M., Zhan, W., Sieck, G.C., Mantilla, C.B., 2012. Reduced ribosomal protein S6 phosphorylation following progressive resistance exercise in growing adolescent rats. J. Strength Cond. Res. 26 (6), 1657-1666.

Hettwer, S., Dahinden, P., Kucsera, S., Farina, C., Ahmed, S., Fariello, R., Drey, M., Sieber, C.C., Vrijbloed, J.W., 2013. Elevated levels of a C-terminal agrin fragment identifies a new subset of sarcopenia patients. Exp. Gerontol. 48, 68-75.

Hildreth, K.L., Barry, D.W., Moreau, K.L., Vande Griend, J., Meacham, R.B., Nakamura, T., Wolfe, P., Kohrt, W.M., Ruscin, J.M., Kittelson, J., Cress, M.E., Ballard, R., Schwartz, R.S., 2013. Effects of testosterone and progressive resistance exercise in healthy, highly functioning older men with low-normal testosterone levels. J. Clin. Endocrinol. Metab. 98 (5), 1891-1900. http://dx.doi.org/10.1210/jc.2012-3695.

Hornberger, T.A., Farrar, R.P., 2004. Physiological hypertrophy of the FHL muscle following 8 weeks of progressive resistance exercise in the rat. Can. J. Appl. Physiol. 29 (1), 16-31.

Ibebunjo, C., Chick, J.M., Kenadall, T., Eash, J.K., Li, C., Zhang, Y., Vickers, C., Wu, Z., Clarke, B.A., Shi, J., Cruz, J., Fournier, B., Brachat, S., Gutzwiller, S., Ma, Q., Markovits, J., Broome, M., Steinkrauss, M., Skuba, E., Galarneau, J., Gygi, S.P., Glass, D.J., 2012. Genomic and proteomic profiling reveals reduced mitochondrial function and disruption of the neuromuscular junction driving rat sarcopenia. Mol. Cell. Biol. Isidori, A.M., Giannetta, E., Greco, E.A., Gianfrilli, D., Bonifacio, V., Isidori, A., Lenzi, A., Fabbri, A., 2005. Effects of testosterone on body composition, boné metabolism and sérum lipid profile in middle-aged men: a meta-analysis. Clin. Endocrinol. 63 , 280-293.

Jang, Y.C., Van Remmen, H., 2011. Age-associated alterations of neuromuscular junction. Exp. Gerontol. 46 (2-3), 193-198.

Katznelson, L., Robinson, M.W., Coyle, C.L., Lee, H., Farrell, C.E., 2006. Effect of modest testosterone supplementation and exercise for 12 weeks on body composition and quality of life in elderly men. Eur. J. Endocrinol. 155, 867-875.

Kawada, S., Okuno, M., Ishii, N., 2006. Testosterone causes decrease in the content of skeletal muscle myostatin. Int. J. Sports Health Sci. 4, 44-48.

Kovacheva, E.L., Sinha-Hikim, A.P., Shen, R., Sinha, I., Sinha-Hikim, I., 2010 Testosterone supplementation reverses sarcopenia in aging through regulation of myostatin, c-Jun NH2-terminal kinase, notch, and Akt signaling pathways. Endocrinology 151, 628-638.

Kraemer, W.J., Flanagan, S.D., Volek, J.S., Nindl, B.C., Vingren, J.L., Dunn-Lewis, C., Comstock, B.A., Hooper, D.R., Szivak, T.K., Looney, D.P., Maresh, C.M., Hymer, W.C., 2013. Resistance exercise induces region-specific adaptations in anterior pituitary gland structure and function in rats. J. Appl. Physiol. 115, 1641-1647.

Krause Neto, W., Gama, E.F., 2013. The process of aging and neuromuscular junction morphology of limb muscles: a systematic review. J. Morphol. Sci. 30 (4), 213-218.

Krause Neto, W., Gama, E.F., Rocha, L.Y., Ramos, C.C., Taets, W., Scapini, K.B., Ferreira, J.B., Rodrigues, B., Caperuto, E., 2015. Effects of testosterone on lean mass gain in elderly men: systematic review with meta-analysis of controlled and randomized studies. Age (Dordr.) 37 (1), 9742.

Krause Neto, W., Silva, W.A., Ciena, A.P., Anaruma, C.A., Gama, E.F., 2016. Vertical climbing for rodent resistance training: a discussion about training parameters. Int. J. Sports Sci. 6 (1A), 36-49.

Kvorning, T., Christensen, L.L., Madsen, K., Nielsen, J.L., Gejl, K.D., Brixen, K., Andersen, M., 2013. Mechanical muscle function and lean body mass during supervised strength training and testosterone therapy in aging men with low-normal testosterone levels. J. Am. Geriatr. Soc. 61 (6), 957-962. http://dx.doi.org/10.1111/jgs.12279.

Lambert, C.P., Sullivan, D.H., Freeling, S.A., Lindquist, D.M., Evans, W.J., 2002. Effects of testosterone replacement and/or resistance exercise on the composition of megestrol acetate stimulated weight gain in elderly men: a randomized controlled trial. J. Clin. Endocrinol. Metab. 87 (5), 2100-2106.

Lee, S., Barton, E.R., Sweeney, H.L., Farrar, R.P., 2004. Viral expression of insulin-like growth factor-I enhances muscle hypertrophy in resistance-trained rats. J. Appl. Physiol. 96 (3), 1097-1104.

Li, Y., Lee, Y.I., Thompson, W.J., 2011. Changes in aging mouse neuromuscular junctions are explained by degeneration and regeneration of muscle fiber segments at the synapse. J. Neurosci. 31 (42), 14910-14919.

Luo, L., Lu, A., Wang, Y., Hong, A., Chen, Y., Hu, J., Li, X., Qin, Z., 2013. Chronic resistance training activates autophagy and reduces apoptosis of muscle cells by modulating IGF-1 and its receptors, Akt/mTOR and Akt/FOXO3a signaling in aged rats. Exp. Gerontol. 48, 427-436.

Lynch, G.S., Schertzer, J.D., Ryall, J.G., 2007. Therapeutic approaches for muscle wasting disorders. Pharmacol. Ther. 113, 461-487.

Mackey, A.L., Esmarck, B., Kadi, F., Koskinen, S.O., Kongsgaard, M., Sylvestersen, A., Hansen, J.J., Larsen, G., Kjaer, M., 2007. Enhanced satellite cell proliferation with resistance training in elderly men and women. Scand. J. Med. Sci. Sports 17, 34-42.

Monks, D.A., O'Bryant, E.L., Jordan, C.L., 2004. Androgen receptor immunoreactivity in skeletal muscle: enrichment at the neuromuscular junction. J. Comp. Neurol. 473, 59-72.

Nagaya, N., Herrera, A.A., 1995. Effects of testosterone on synaptic efficacy at neuromuscular junctions in a sexually dimorphic muscle of male frogs. J. Physiol. 483, $141-153$.

Nudler, S.I., Pagani, M.R., Urbano, F.J., McEnery, M.W., Uchitel, O.D., 2005. Testosterone modulates $\mathrm{Ca}_{\mathrm{v} 2.2}$ calcium channels functional expression at rat levator ani neuromuscular junction. Neuroscience 134, 817-826.

Olsen, S., Aagaard, P., Kadi, F., Tufekovic, G., Verney, J., Olesen, J.L., Suetta, C., Kjaer, M., 2006. Creatine supplementation augments the increase in satellite cell and myonuclei in human skeletal muscle induced by strength training. J. Physiol. 573, 525-534.

Peterson, M.D., Rhea, M.R., Sen, A., Gordon, P.M., 2010. Resistance exercise for muscle strength in older adults: a meta-analysis. Ageing Res. Rev. 9, 226-237.

Sakita, M., Murakami, S., FUJINO, H., 2016. Age-related morphological regression of myelinated fibers and capillary architecture of distal peripheral nerves in rats. BMC Neurosci. 17 (1), 39.

Sinha-Hikim, I., Artaza, J., Woodhouse, L., Gonzales-Cadavid, N., Singh, A.B., Lee, M.I., Storer, T.W., Casaburi, R., Shen, R., Bhasin, S., 2002. Testosterone-induced increase in muscle size in health young men is associated with muscle fiber hypertrophy. Am. J. Physiol. Endocrinol. Metab. 283, E154-E164.

Sinha-Hikim, I., Cornford, M., Gaytan, H., Lee, M.L., Bhasin, S., 2006. Effects of testosterone supplementation on skeletal muscle fiber hypertrophy and satellite cells in community-dwelling older men. J. Clin. Endocrinol. Metab. 91 (8), 3024-3033.

Snijders, T., Verdijk, L.B., Van Loon, L.J.C., 2009. The impact of sarcopenia and exercise training on skeletal muscle satellite cells. Ageing Res. Rev. 8, 328-338.

Tanasov, V.S., Neto, W.K., Gonçalves, L., Maifrino, L.B.M., De Souza, R.R., Gama, E.F., 2014. Use of anabolic steroid altered the liver morphology of rats. Int. J. Morphol. 32 (3), 756-760.

Tauchi, H., Yoshida, T., Kobayashi, H., 1971. Age change of skeletal muscle of rats. Gerontologia 17, 219-227.

Trenerry, M.K., Carey, K.A., Ward, A.C., Cameron-Smith, D., 2007. STAT3 signaling is activated in human skeletal muscle following acute resistance exercise. J. Appl. 
Physiol. 102, 1483-1489.

Trenerry, M.K., Della Gatta, P.A., Larsen, A.E., Garnham, A.P., Cameron-Smith, D., 2011. Impact of resistance exercise training on interleukin-6 and JAK/STAT in young men. Muscle Nerve 43, 385-392.

Verdijk, L.N., Koopman, R., Schaart, G., Meijer, K., Savelberg, H.C.M., Van Loon, L.J.C., 2007. Satellite cell content is specifically reduced in type II skeletal muscle fibers in the elderly. Am. J. Physiol. Endocrinol. Metab. 292, E151-E157.

Verney, J., Kadi, F., Charifi, N., Feasson, L., Saafi, M.A., Castells, J., Piehl-Aulin, K., Denis, C., 2008. Effects of combined lower body endurance and upper body resistance training on the satellite cell pool in elderly subjects. Muscle Nerve 38, 1147-1154. Willoughby, D.S., Taylor, L., 2004. Effects of sequential bouts of resistance exercise on androgen receptor expression. Med. Sci. Sports Exerc. 36, 1499-1506.

Woodhouse, L.J., Reisz-Porszasz, S., Javanbakht, M., Storer, T.W., Lee, M., Zerounian, H., Bhasin, S., 2003. Development of models to predict anabolic response to testosterone administration in healthy young men. Am. J. Physiol. Endocrinol. Metab. 284, E1009-E1017.

Yarasheski, K.E., Lemon, P.W.R., Gilloteaux, J., 1990. Effect of heavy-resistance exercise training on muscle fiber composition in young rats. J. Appl. Physiol. 69 (2), 434-437. 